\title{
Neighbourhood disadvantage and depressive symptoms among adolescents followed into emerging adulthood
}

\author{
Rise B Goldstein, ${ }^{\circ}$ Awapuhi K Lee, ${ }^{1,2}$ Denise L Haynie, ${ }^{1}$ Jeremy W Luk, 1,3 \\ Brian J Fairman, ${ }^{1}$ Danping Liu, ${ }^{4}$ Jacob S Jeffers, ${ }^{1}$ Bruce G Simons-Morton, \\ Stephen E Gilman ${ }^{1,5}$
}

For numbered affiliations see end of article.

\section{Correspondence to}

Dr Rise B Goldstein, Social and Behavioral Sciences

Branch, Division of Intramural Population Health Research,

Eunice Kennedy Shriver National Institute of Child Health and Human Development, Bethesda, MD 20892, USA; goldster@ mail.nih.gov

Received 12 December 2018 Revised 11 February 2019 Accepted 9 March 2019

Published Online First

30 March 2019
Check for updates

(C) Author(s) (or their employer(s)) 2019. No commercial re-use. See rights and permissions. Published by BMJ.

To cite: Goldstein RB,

Lee AK, Haynie DL, et al. J

Epidemiol Community Health 2019:73:590-597.

\begin{abstract}
Background Residents of disadvantaged neighbourhoods report higher levels of depressive symptoms; however, few studies have employed prospective designs during adolescence, when depression tends to emerge. We examined associations of neighbourhood social fragmentation, income inequality and median household income with depressive symptoms in a nationally representative survey of adolescents.

Methods The NEXT Generation Health Study enrolled 10th-grade students from 81 US high schools in the 2009-2010 school year. Depressive symptoms were assessed with the Modified Depression Scale (wave 1) and the paediatric Patient-Reported Outcome Measurement Information System (waves 2-6). Neighbourhood characteristics at waves 1, 3, 4, and 5 were measured at the census tract level using geolinked data from the American Community Survey 5-year estimates. We used linear mixed models to relate neighbourhood disadvantage to depressive symptoms controlling for neighbourhood and individual sociodemographic factors.
\end{abstract}

Results None of the models demonstrated evidence for associations of social fragmentation, income inequality or median household income with depressive symptoms.

Conclusion Despite the prospective design, repeated measures and nationally representative sample, we detected no association between neighbourhood disadvantage and depressive symptoms. This association may not exist or may be too small to detect in a geographically dispersed sample. Given the public health significance of neighbourhood effects, future research should examine the developmental timing of neighbourhood effects across a wider range of ages than in the current sample, consider both objective and subjective measures of neighbourhood conditions, and use spatially informative techniques that account for conditions of nearby neighbourhoods.

\section{INTRODUCTION}

The neighbourhood environment appears to be an important determinant of mental health. ${ }^{1}$ Neighbourhood attributes linked to depression include socioeconomic disadvantage, instability, lack of social cohesion and income inequality. ${ }^{2-4}$ Social theories (eg, social ecologic, social cognitive and social stress) ${ }^{5}$ suggest that associations of neighbourhood attributes with depression arise from the lack of investment and limited resources for health-promoting behaviours in disadvantaged neighbourhoods. Resource constraints break down social processes at the aggregate (eg, through low social cohesion) and individual (eg, through breaking social ties) ${ }^{6-10}$ levels that benefit mental well-being. ${ }^{5}$ Associations of low neighbourhood income with depression may arise because of increased exposure to interpersonal violence and other stressful life events in contexts without sufficient social and material supports to buffer their effects, ${ }^{11}$ and high income inequality generates invidious social comparisons which are deleterious for mental health. ${ }^{12}$ Associations of neighbourhood income or income inequality with depression may also exist because of higher social fragmentation or lower social cohesion in more disadvantaged, less egalitarian places. ${ }^{13} 14$

Neighbourhood economic disadvantage is captured by median household income and percentages of residents below the poverty line, with less than high school education, unemployed and receiving public assistance. We found 26 studies showing that residents of neighbourhoods with higher economic disadvantage had higher scores on depressive symptom scales and higher risks of major depressive disorder (eg, ORs 1.05-2.40). ${ }^{34}$ Residents of neighbourhoods with higher income inequality also had higher levels of depressive symptoms. ${ }^{121516}$ However, there were 19 studies in which neighbourhood disadvantage was not associated with depression.

In 12 studies, residents of socially disadvantaged neighbourhoods (ie, characterised by residential instability or low social cohesion) had higher mean levels of depressive symptoms and higher risks for clinical depression; conversely, residents of neighbourhoods with lower social disadvantage (eg, greater social cohesion) had lower scores and lower risks. In other studies, however, neighbourhood social disadvantage was not associated with residents' depression.

These inconsistent findings may reflect methodological differences between studies such as prospective versus cross-sectional study designs, focus on single regions or population subgroups rather than nationally representative samples, sample size, length of follow-up, definition of neighbourhood (census tract or ZIP code vs respondent-defined 

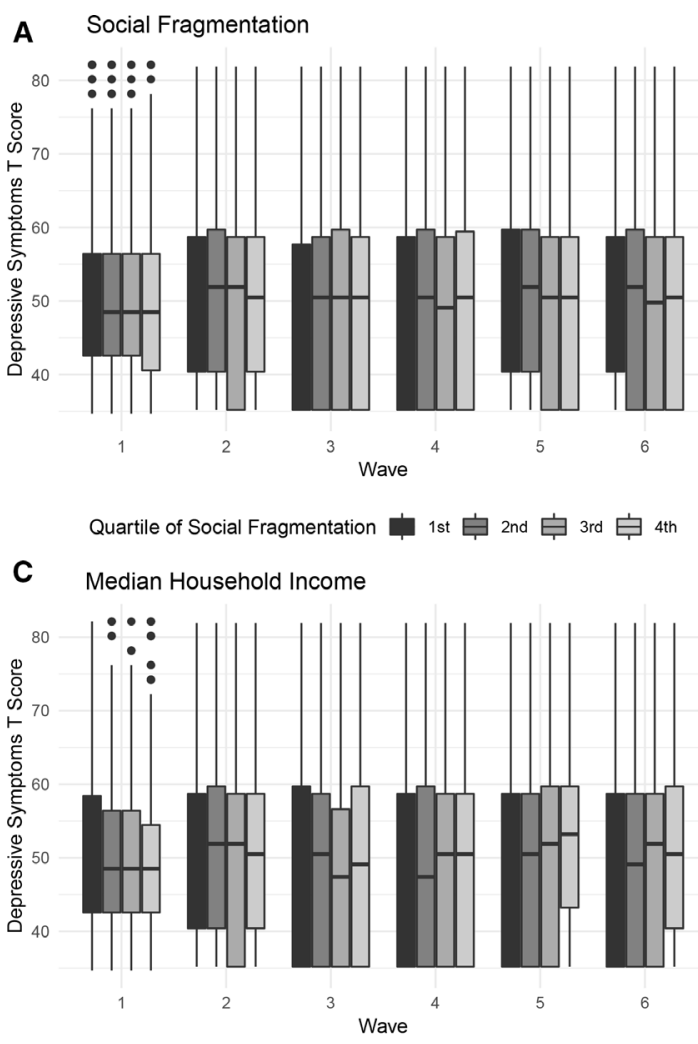

Quartile of Neighborhood Household Income 1st 穴 2nd 官 3rd 由 4th
B Income Inequality

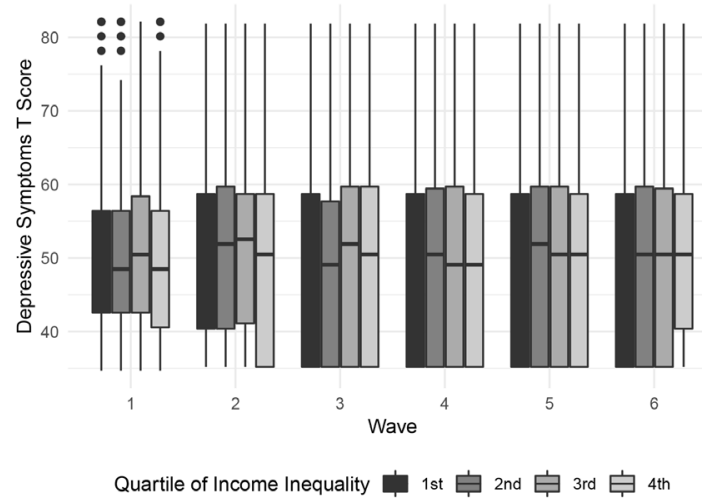

neighbourhood boundaries), assessment of disadvantage (Census data vs respondents' perceptions) and measurement of depression. Prospective studies with longer follow-up periods were less likely to detect associations of neighbourhood social or economic disadvantage with depression. The studies reporting associations were based on follow-up periods $<5$ years. ${ }^{3417}$ Of the studies reporting no associations between neighbourhood context and depression, nine had follow-up periods $\geq 5$ years and five followed up respondents for $\geq 10$ years. ${ }^{3417-20}$ Notably, few studies with follow-up periods $>5$ years included repeated measures of neighbourhood exposures, which could fail to detect associations if neighbourhood effects decay over time.

Neighbourhood studies of mental health, most of which focused on adults or young children, ${ }^{3417}$ may also have missed the developmental period of greatest risk, as depression tends to emerge during adolescence. ${ }^{21} 22$ Pabayo et al reported an association between higher income inequality and depressive symptoms among adolescent girls but not boys. ${ }^{12}$ In contrast, Airaksinen et al found that neighbourhood socioeconomic conditions were not associated with depressive symptoms measured repeatedly over five waves in young adulthood, though neighbourhood conditions were only assessed at baseline. ${ }^{18}$ Similarly, in a 14-year study of depressive symptoms among US adolescents, Barr found that Census-based neighbourhood socioeconomic conditions at baseline were unrelated to depression, whereas participants' perceptions of neighbourhood safety and neglect were associated with higher depressive symptoms. ${ }^{19}$ However, the use of subjective measures is problematic if individuals with depression perceive their neighbourhoods more negatively than individuals without depression. ${ }^{23} 24$
Because of these inconsistent findings, we examined prospective associations of three features of neighbourhood conditions with depressive symptoms in a nationally representative sample of adolescents: social fragmentation, neighbourhood income inequality and median household income. We leveraged the following design strengths of the NEXT Generation Health Study ('NEXT') $)^{25}$ : (1) a nationally representative sample; (2) six annual follow-up assessments providing repeated measures of depressive symptoms through young adulthood; and (3) repeated measurement of neighbourhood exposures utilising objective, Census-derived neighbourhood characteristics geolinked to respondents' addresses at four study waves. We hypothesised that higher social fragmentation, lower median household income and higher income inequality would be associated with higher levels of depressive symptoms between ages 16 and 22 .

\section{METHODS}

\section{Sample}

NEXT enrolled a nationally representative sample of adolescents using a three-stage stratified design targeting 10th graders enrolled in public, private or parochial high schools in the USA in the school year 2009-2010. ${ }^{25}$ Primary sampling units (PSUs, $n=27$ ) consisted of school districts or groups of school districts stratified by US Census divisions. Schools in each PSU with 10th-grade classes were sampled with probability proportional to enrolment; $58.4 \%$ of sampled schools $(n=81)$ participated. All students within randomly selected classrooms (1 to 5 per school) were eligible to participate. Parents provided informed consent for their children's participation and youth 
Table 1 Sociodemographic characteristics of NEXT respondents (total N=2752) and their residential neighbourhoods by survey wave, $\%$ or mean $(\mathrm{SE})^{\star}$

\begin{tabular}{|c|c|c|c|c|c|c|}
\hline Characteristic & $\begin{array}{l}\text { Wave } 1 \\
(n=2486)\end{array}$ & $\begin{array}{l}\text { Wave } 2 \\
(n=2388)\end{array}$ & $\begin{array}{l}\text { Wave } 3 \\
(n=2354)\end{array}$ & $\begin{array}{l}\text { Wave } 4 \\
(n=2088)\end{array}$ & $\begin{array}{l}\text { Wave } 5 \\
(n=2118)\end{array}$ & $\begin{array}{l}\text { Wave } 6 \\
(n=2042)\end{array}$ \\
\hline Depressive symptom $T$-score & $50.7(0.4)$ & $51.2(0.5)$ & $50.5(0.6)$ & $50.4(0.4)$ & $51.2(0.4)$ & $50.9(0.4)$ \\
\hline \multicolumn{7}{|l|}{ Neighbourhood } \\
\hline \multicolumn{7}{|l|}{ Social Fragmentation Indext } \\
\hline Lowest quartile & $33.2(4.9)$ & $32.2(5.3)$ & $32.6(5.6)$ & $35.9(3.7)$ & $36.3(4.2)$ & $33.9(4.6)$ \\
\hline Second quartile & $43.8(6.3)$ & $43.4(6.2)$ & $40.4(6.1)$ & $29.2(3.2)$ & $32.2(3.6)$ & $32.3(3.8)$ \\
\hline Third quartile & $14.3(4.2)$ & $15.6(4.5)$ & $17.2(4.5)$ & $18.4(3.0)$ & $18.4(2.7)$ & $19.9(3.0)$ \\
\hline Highest quartile & $8.8(3.7)$ & $8.9(3.6)$ & $9.9(3.7)$ & $16.5(2.4)$ & $13.0(2.8)$ & $13.8(3.0)$ \\
\hline \multicolumn{7}{|l|}{ Gini coefficient of income inequality $\dagger$} \\
\hline Lowest quartile & $29.5(5.7)$ & $29.6(5.8)$ & $27.8(5.3)$ & $28.3(3.8)$ & $29.4(3.4)$ & $28.8(3.5)$ \\
\hline Second quartile & $25.0(2.5)$ & $24.2(2.4)$ & $25.4(2.9)$ & $24.7(2.7)$ & $26.8(2.9)$ & $25.6(3.0)$ \\
\hline Third quartile & $25.6(4.3)$ & $24.8(4.1)$ & $23.9(3.5)$ & $24.9(3.8)$ & $26.1(3.0)$ & $26.5(3.1)$ \\
\hline Highest quartile & $19.9(5.3)$ & $21.4(5.6)$ & $23.0(5.4)$ & $22.2(2.0)$ & $17.7(2.9)$ & $19.1(3.1)$ \\
\hline \multicolumn{7}{|l|}{ Median household incomet } \\
\hline Lowest quartile & $20.1(5.6)$ & $21.3(5.8)$ & $20.7(5.2)$ & $23.3(2.8)$ & $20.7(2.4)$ & $22.4(3.0)$ \\
\hline Second quartile & $18.0(3.6)$ & $17.6(3.4)$ & $20.2(3.2)$ & $20.9(2.5)$ & $21.0(2.6)$ & $22.3(3.0)$ \\
\hline Third quartile & $30.1(4.9)$ & $29.5(4.8)$ & $28.6(4.6)$ & $30.2(3.1)$ & $29.1(3.2)$ & $26.4(3.3)$ \\
\hline Highest quartile & $31.8(5.5)$ & $31.6(5.7)$ & $30.5(5.5)$ & $25.6(3.6)$ & $29.2(4.3)$ & $28.9(4.3)$ \\
\hline \multicolumn{7}{|l|}{ Percentage of minority residents $†$} \\
\hline Lowest quartile & $33.0(5.5)$ & $32.8(5.8)$ & $31.6(5.7)$ & $36.1(4.6)$ & $34.0(4.7)$ & $32.1(5.0)$ \\
\hline Second quartile & $44.9(6.9)$ & $43.4(6.8)$ & $43.7(6.8)$ & $38.8(4.9)$ & $42.4(5.7)$ & $40.3(6.0)$ \\
\hline Third quartile & $13.5(3.6)$ & $12.7(3.5)$ & $13.6(3.6)$ & $14.3(3.3)$ & $14.2(3.2)$ & $15.3(3.3)$ \\
\hline Highest quartile & $8.6(4.5)$ & $11.1(5.2)$ & $11.1(5.2)$ & $10.9(3.2)$ & $9.4(3.2)$ & $12.3(4.2)$ \\
\hline \multicolumn{7}{|l|}{ Respondent/Family } \\
\hline Sex (\% male) & $45.6(1.7)$ & $44.8(1.8)$ & $44.8(1.6)$ & $41.2(2.0)$ & $40.1(1.9)$ & $38.4(1.9)$ \\
\hline Age & $16.3(0.03)$ & $17.2(0.03)$ & $18.2(0.03)$ & $19.2(0.02)$ & $20.3(0.02)$ & $21.3(0.02)$ \\
\hline \multicolumn{7}{|l|}{ Race or ethnicity } \\
\hline Non-Hispanic White & $57.8(5.4)$ & $58.8(6.0)$ & $58.8(6.0)$ & $62.1(5.8)$ & $61.1(5.3)$ & $57.1(6.3)$ \\
\hline Non-Hispanic Black/African-American & $17.6(3.6)$ & $17.3(4.1)$ & $17.1(4.1)$ & $13.5(3.3)$ & $13.6(3.4)$ & $19.7(4.9)$ \\
\hline Hispanic or Latino & $19.7(3.9)$ & $19.5(4.0)$ & $19.9(3.9)$ & $19.5(4.3)$ & $19.9(3.8)$ & $18.7(3.8)$ \\
\hline Other & $5.0(1.1)$ & $4.3(1.0)$ & $4.1(0.8)$ & $4.9(1.0)$ & $5.4(1.0)$ & $4.5(1.1)$ \\
\hline \multicolumn{7}{|l|}{ Family affluence } \\
\hline Low & $23.9(2.7)$ & $23.0(2.9)$ & $23.0(3.1)$ & $22.2(2.7)$ & $22.5(2.6)$ & $22.4(3.1)$ \\
\hline Moderate & $48.8(1.5)$ & $49.8(1.2)$ & $49.0(1.5)$ & $48.3(1.8)$ & $49.2(1.5)$ & $49.5(1.6)$ \\
\hline High & $27.3(2.5)$ & $27.2(2.5)$ & $28.0(2.8)$ & $29.4(2.7)$ & $28.4(2.6)$ & $28.1(2.7)$ \\
\hline
\end{tabular}

*Wave-specific percentages for some variables do not add to $100 \%$ because of rounding.

†Neighbourhood measures at each wave were standardised to a mean of 0 and SD of 1 in all US census tracts.

provided assent (if $<18$ years of age) and consent once they reached 18 years of age. The protocol including informed consent procedures was approved by the institutional review board of the Eunice Kennedy Shriver National Institute of Child Health and Human Development and conforms to the principles embodied in the Declaration of Helsinki.

Among eligible students, $73.4 \%(n=2786)$ participated. Baseline surveys were administered in 2009-2010; however, the timing of school approval for participation resulted in the collection of baseline data for 260 respondents during wave 2 20102011,11 th grade). This study used data from the first six annual waves that were self-administered either in school or online. Retention rates were $86.8 \%$ at wave $2,83.9 \%$ at wave $3,75.9 \%$ at wave $4,76.6 \%$ at wave 5 and $79.9 \%$ at wave 6 . Schools with large percentages of African American students were oversampled to obtain reliable estimates for them.

\section{Measures}

\section{Depressive symptoms}

We assessed depressive symptoms at wave 1 using the Modified Depression Scale (MDS). ${ }^{26}$ The MDS asks respondents to rate on a Likert scale from 'never' to 'always', the frequency with which they experienced symptoms such as sadness, grouchiness or irritability, and increases or decreases in appetite and sleep over the past 30 days (Cronbach's $\alpha=0.76-0.80) .^{26-28}$

At waves 2-6, we measured depressive symptoms using the paediatric Patient Reported Outcome Measurement Information System $\left(\mathrm{PROMIS}^{29}\right.$ ) scale. This scale asks respondents to rate on a Likert scale from 'never' to 'almost always', ${ }^{30}$ the frequency with which they experienced symptoms including feeling that they cannot do anything right, feeling that everything in their lives had gone wrong and being unable to stop feeling sad over the preceding 7 days (Cronbach's $\alpha=0.85$ and test-retest 
Table 2 Regression coefficients (95\% Cls) from linear mixed models of depressive symptoms in the NEXT Generation Health Study, waves 1-6 $(n=2752)$

\begin{tabular}{|c|c|c|c|c|c|}
\hline Characteristic & Age-adjusted* & Model 1† & Model 2 $\ddagger$ & Model $3 \S$ & Model 4 ๆ \\
\hline \multicolumn{6}{|l|}{ Neighbourhood } \\
\hline \multicolumn{6}{|l|}{ Gini coefficient of income inequality ${ }^{* *}+\dagger$} \\
\hline Lowest quartile & Referent & Referent & Referent & Referent & Referent \\
\hline Second quartile & $0.17(-0.35$ to 0.69$)$ & $0.10(-0.43$ to 0.64$)$ & $0.10(-0.45$ to 0.64$)$ & $0.10(-0.45$ to 0.66$)$ & $0.15(-0.43$ to 0.73$)$ \\
\hline Third quartile & $-0.23(-0.79$ to 0.33$)$ & $-0.33(-0.90$ to 0.23$)$ & $-0.41(-1.00$ to 0.17$)$ & $-0.38(-0.98$ to 0.21$)$ & $-0.34(-0.96$ to 0.28$)$ \\
\hline Highest quartile & $-0.34(-0.93$ to 0.24$)$ & $-0.43(-1.02$ to 0.16$)$ & $-0.56(-1.18$ to 0.05$)$ & $-0.46(-1.14$ to 0.22$)$ & $-0.43(-1.14$ to 0.29$)$ \\
\hline$F, d f=3(P)$ & $1.3(0.26)$ & $1.7(0.18)$ & $2.4(0.07)$ & $1.6(0.18)$ & $1.6(0.18)$ \\
\hline \multicolumn{6}{|l|}{ Median household income ${ }^{* *} \dagger \dagger$} \\
\hline Lowest quartile & $-0.31(-0.92$ to 0.30$)$ & $-0.46(-1.09$ to 0.17$)$ & $-0.54(-1.20$ to 0.11$)$ & $-0.30(-1.01$ to 0.42$)$ & $-0.27(-1.19$ to 0.64$)$ \\
\hline Second quartile & $0.10(-0.51$ to 0.72$)$ & 0.03 (-0.60 to 0.65$)$ & $-0.06(-0.68$ to 0.57$)$ & $0.03(-0.63$ to 0.69$)$ & 0.06 (-0.68 to 0.80$)$ \\
\hline Third quartile & $-0.26(-0.81$ to 0.30$)$ & $-0.28(-0.86$ to 0.31$)$ & $-0.36(-0.92$ to 0.20$)$ & $-0.29(-0.87$ to 0.28$)$ & $-0.27(-0.88$ to 0.34$)$ \\
\hline Highest quartile & Referent & Referent & Referent & Referent & Referent \\
\hline$F, d f=3(P)$ & $1.0(0.39)$ & $1.3(0.27)$ & $1.5(0.22)$ & $0.8(0.50)$ & $0.7(0.53)$ \\
\hline \multicolumn{6}{|l|}{ Social Fragmentation Index** $\dagger \dagger$} \\
\hline Lowest quartile & Referent & Referent & & & Referent \\
\hline Second quartile & $0.22(-0.28$ to 0.72$)$ & $0.12(-0.40$ to 0.64$)$ & & & $0.06(-0.54$ to 0.66$)$ \\
\hline Third quartile & $-0.32(-0.95$ to 0.31$)$ & $-0.49(-1.16$ to 0.18$)$ & & & $-0.56(-1.39$ to 0.27$)$ \\
\hline Highest quartile & $0.17(-0.52$ to 0.87$)$ & 0.03 (-0.75 to 0.82$)$ & & & 0.06 (-0.95 to 1.07$)$ \\
\hline$F, d f=3(P)$ & $1.3(0.27)$ & $1.6(0.20)$ & & & $1.7(0.17)$ \\
\hline \multicolumn{6}{|l|}{ Percentage of minority residents ${ }^{* *}+\dagger$} \\
\hline Lowest quartile & Referent & Referent & & Referent & Referent \\
\hline Second quartile & 0.54 (0.02 to 1.06$)$ & $0.45(-0.07$ to 0.97$)$ & & 0.61 (0.07 to 1.16$)$ & 0.66 (0.09 to 1.23$)$ \\
\hline Third quartile & $0.66(-0.06$ to 1.37$)$ & $0.48(-0.31$ to 1.26$)$ & & $0.71(-0.08$ to 1.50$)$ & 0.88 (0.01 to 1.74$)$ \\
\hline Highest quartile & $0.19(-0.63$ to 1.01$)$ & $-0.07(-1.00$ to 0.86$)$ & & $0.14(-0.79$ to 1.07$)$ & $0.32(-0.66$ to 1.31$)$ \\
\hline $\mathrm{F}, \mathrm{df}=3(\mathrm{P})$ & $2.0(0.12)$ & $1.7(0.16)$ & & $2.6(0.05)$ & $2.5(0.06)$ \\
\hline \multicolumn{6}{|l|}{ Respondent/Family } \\
\hline Age, per year** & & & & $-0.08(-0.17$ to 0.00$)$ & $-0.08(-0.16$ to 0.01$)$ \\
\hline Male sex & $-4.95(-5.62$ to -4.29$)$ & & & $-4.94(-5.61$ to -4.27$)$ & $-4.95(-5.62$ to -4.28$)$ \\
\hline \multicolumn{6}{|l|}{ Race or ethnicity } \\
\hline Non-Hispanic White & Referent & & & Referent & Referent \\
\hline Non-Hispanic Black/African American & $0.83(-0.13$ to 1.80$)$ & & & $0.38(-0.68$ to 1.43$)$ & $0.41(-0.73$ to 1.55$)$ \\
\hline Hispanic or Latino & $0.18(-0.72$ to 1.09$)$ & & & $0.17(-0.84$ to 1.18$)$ & $0.19(-0.87$ to 1.25$)$ \\
\hline Other & $2.58(0.97$ to 4.19$)$ & & & 2.22 (0.61 to 3.84$)$ & 2.22 (0.58 to 3.86$)$ \\
\hline$F, d f=3(P)$ & $3.9(0.01)$ & & & $2.6(0.05)$ & $2.5(0.06)$ \\
\hline \multicolumn{6}{|l|}{ Family affluence } \\
\hline Low & $0.51(-0.44$ to 1.45$)$ & & & $0.60(-0.33$ to 1.53$)$ & $0.59(-0.35$ to 1.54$)$ \\
\hline Moderate & $-0.09(-0.95$ to 0.77$)$ & & & $0.13(-0.72$ to 0.98$)$ & $0.13(-0.71$ to 0.98$)$ \\
\hline High & Referent & & & Referent & Referent \\
\hline $\mathrm{F}, \mathrm{df}=2(\mathrm{P})$ & $1.1(0.35)$ & & & $1.0(0.38)$ & $0.9(0.40)$ \\
\hline
\end{tabular}

*Each variable is modelled separately, adjusted only for respondent age.

tEach neighbourhood characteristic is analysed in a separate regression adjusted for respondent-level and family-level covariates.

‡Income inequality and median household income are modelled separately, each adjusted for respondent-level/family-level and neighbourhood-level covariates.

§Income inequality and median household income are modelled simultaneously, adjusted for respondent-level/family-level and neighbourhood-level covariates.

IAll neighbourhood, respondent-level and family-level characteristics are entered simultaneously into a single model.

**Age and neighbourhood characteristics are modelled as time-varying.

††All neighbourhood measures at each wave were standardised to a mean of 0 and SD of 1 in all US census tracts.

reliability $=0.76) .{ }^{31}$ The decision to switch from the MDS to the PROMIS reflected accumulating evidence of its desirable psychometric properties such as internal consistency and test-retest reliability and discrimination over wider ranges of depressive severity. ${ }^{30-34}$

Scores on the PROMIS were converted into $T$-scores based on distributions of scores in the general US paediatric population.
We analysed depressive symptom $T$-scores for the PROMIS (mean $=50, \mathrm{SD}=10$ ) and MDS scores standardised to the same mean and SD. The correlation between MDS at wave 1 and PROMIS at wave 2 was 0.48 , very similar to correlations between PROMIS scores at any two consecutive waves $(0.50$ $0.54)$, suggesting the two measures are performing similarly in the NEXT sample and justifying our combining them for analysis. 


\section{Neighbourhood characteristics}

Respondents' home addresses were geocoded to census tracts at waves 1, 3, 4 and 5. Consistent with previous studies of neighbourhood disadvantage and adverse health outcomes, ${ }^{35-39}$ and because of their greater stability compared with single-year estimates, neighbourhood measures were based on 5-year census tract-level estimates from the American Community Survey $\left(\mathrm{ACS}^{40}\right)$ : 2007-2011 for wave 1, 2009-2013 for wave 3, 20102014 for wave 4 and 2011-2015 for wave 5. Neighbourhood characteristics were therefore updated based on respondents' geocoded census tracts at waves 3,4 and 5. Because respondents were not geocoded at waves 2 and 6 , we applied the values of the neighbourhood variables at wave 1 to wave 2 and those at wave 5 to wave 6 . All neighbourhood variables were standardised to a mean of 0 and SD of 1 in the total US population and treated as time-varying in all models. At baseline, there were 1105 census tracts represented in the sample; census tracts were more geographically dispersed than PSUs, with an average of 41 census tracts represented within each PSU.

\section{Social fragmentation}

It is an index consisting of the sum of the standardised percentages in respondents' census tracts of female-headed households, residents living in the area $<5$ years, foreign-born residents and renters. ${ }^{41}$ Single-parent households are significantly more likely than two-parent households to be poor. ${ }^{42}$ Poverty, particularly among single mothers, is a strong risk factor for the lack of social support that might buffer the stress resulting from competing demands of supporting their families financially, parenting and other life tasks. ${ }^{43-45}$ This constellation of adversity may contribute to role overload. ${ }^{46}$ Higher proportions of households in these circumstances may mean fewer adults able to act as long-term, stable, dependable sources of emotional and adaptive social support or consistently enforced norms of prosocial behaviour. ${ }^{647}$ Cultural and linguistic barriers in neighbourhoods with high proportions of immigrants, ${ }^{47} 48$ and the residential instability and turnover that frequently characterise renters, may likewise make it difficult to develop and maintain such ties. ${ }^{174748}$

We assessed income inequality using the Gini Index. ${ }^{49} \mathrm{~A}$ value of 0 denotes perfect income equality, whereas a value of 1 denotes the scenario of all income accruing to one individual. Median household income in the ACS was adjusted for inflation to the final year covered by each relevant 5-year estimate (eg, 2014 for wave 4) using the Consumer Price Index. ${ }^{50}$ To enable the assessment of potentially non-linear associations between neighbourhood variables and depressive symptoms, we categorised the neighbourhood variables into quartiles of their distributions in the study sample for analysis.

\section{Covariates}

Time-varying covariates adjusted for in the analyses were minority composition of the neighbourhood (proportion non-White) and respondent age. Respondent-level covariates ascertained at baseline were sex, race/ethnicity (Hispanic/Latino, Black/African-American, White, other), and family socioeconomic status measured using Health Behaviour School-Aged Family Affluence Scale. ${ }^{51}$ This scale is the summed score of four items querying family car $(0,1,2+)$ and computer $(0,1,2+)$ ownership, the past-year frequency of family vacations $(0,1$, $2+)$ and whether respondents had their own bedrooms $(0=$ no, $1=$ yes). Scores ranged from 0 to 7 and were categorised as low $(0-4)$, moderate (5-6) or high (7).

\section{Analytical approach}

Respondents successfully geocoded to census tracts who provided data on sex, family affluence, race/ethnicity, age and at least one measurement of depressive symptoms between waves 1 and 6 of the study were included in the analysis sample $(n=2752)$. Among survey respondents at each wave, 18 of 2524 were missing geocodes at wave 1, 3 of 2395 at wave 3, 30 of 2177 at wave 4 and 6 of 2202 at wave 5 .

We fit linear mixed models with random intercepts for PSUs and individual respondents nested within PSUs to account for the non-independence of respondents sampled from the same PSU and within-person correlation over time. The first set of models examined associations of each covariate with depressive symptoms adjusted only for respondent age. Next, we fit separate multivariable models examining each neighbourhood exposure adjusted for respondent-level covariates (model 1). As associations of neighbourhood income and income inequality may be due in part to differences in social fragmentation across neighbourhoods, we fit models with income only, income inequality only (model 2) and both (model 3), followed by a model that added social fragmentation (model 4), each adjusted for respondent-level and neighbourhood-level covariates. All analyses incorporated NEXT's sampling weights and were performed using SAS V.9.4.

\section{RESULTS}

The mean age of respondents at enrolment was 16.3 years. Forty-six per cent of the sample was men; $55.7 \%$ self-identified as non-Hispanic White, $20.2 \%$ as non-Hispanic Black or African-American, $19.3 \%$ as Hispanic and $4.8 \%$ as another race or ethnicity. Almost half (48.6\%) reported moderate family affluence. Over the six survey waves, there were minimal changes in respondent depressive symptoms, family affluence, race/ ethnicity, and neighbourhood income inequality, percentage of minority residents and median household income (table 1). However, the percentages of male respondents decreased and residents of more socially fragmented neighbourhoods increased over time.

Distributions of depressive symptom $T$-scores by quartiles of neighbourhood exposures within each wave are shown in figure 1 . The distribution of depressive symptoms was virtually the same across quartiles of neighbourhood social fragmentation, median income, and income inequality.

Results of linear mixed models of depressive symptoms are shown in table 2 .

None of the models demonstrated evidence for associations of social fragmentation, income inequality, or median household income with depressive symptoms. For example, in the final regression model, there was no difference in mean depressive symptoms between residents of neighbourhoods at the highest vs lowest quartiles of disadvantage: 0.06 for social fragmentation (95\% CI -0.95 to 1.07$) ;-0.43$ for income inequality $(95 \% \mathrm{CI}$ -1.14 to 0.29 ) and -0.27 for median income $(95 \%$ CI -1.19 to 0.64$)$.

\section{DISCUSSION}

We used repeated annual measures from a prospective study of 2752 respondents enrolled in 10th grade to examine associations of census-based indicators of neighbourhood social and economic disadvantage with depressive symptoms from mid-adolescence into emerging adulthood. We found no evidence of associations of social fragmentation, income inequality or 
median household income of respondents' neighbourhoods and their levels of depressive symptoms.

Our study incorporated design strengths long advocated by neighbourhood researchers: prospective follow-up, population-based sample not limited to a single geographic area, and objective, repeated assessments of neighbourhood characteristics. Moreover, our study was conducted during a developmentally sensitive period for depression and used reliable measures of depressive symptoms. Given these strengths, our findings cast doubt on the existence of robust relationships between neighbourhood social and economic disadvantage and depression among adolescents and emerging adults. These findings are compatible with those reported by many but not all studies with follow-up periods $>5$ years, ${ }^{341752}$ and specifically with those reported by Airaksinen et $a l^{18}$ and Barr ${ }^{19}$ that followed young people into adulthood. Although adolescence and emerging adulthood are important developmental phases for depression, our results, together with those of previous studies, ${ }^{3} 41752$ suggest that neighbourhood structural characteristics may be more important during other phases. Alternatively, adolescents' individual, family or contextual factors during the period captured by the study that operated more proximally to the young people than their residential neighbourhoods, which we were unable to measure, may have obscured any influences of their neighbourhoods. Additionally, although neighbourhood effects may decay over time, there may also be lagged effects over longer intervals than our study could capture.

Barr $^{19}$ found that associations between neighbourhood disadvantage and depressive symptoms disappeared after respondent, parent and interviewer perceptions of neighbourhood safety and physical neglect were accounted for. If individuals do not perceive their neighbourhoods as disadvantaged, neighbourhoods with objectively disadvantageous structural characteristics may not be associated with a higher risk for depression. Adolescents and young adults may be less likely to perceive their neighbourhoods as disadvantaged than older individuals, even in the presence of objective indicators, if their peers in similar neighbourhoods also do not perceive their neighbourhoods as disadvantaged. ${ }^{53}$ Alternatively, supportive relationships with peers may buffer the stressors associated with neighbourhood adversity that are implicated in the aetiology of depression. ${ }^{53}$

Potential study weaknesses include the use of two different measures of depressive symptoms with two different reporting periods and two different underlying metrics over the course of the study. However, results did not change when we reanalysed the data using only the outcomes measured by the PROMIS. Short scales of depressive symptoms may not be sufficiently sensitive for detecting small to moderate neighbourhood effects. The lack of neighbourhood data from waves 2 and 6 is also a potential concern; our inability to update neighbourhood data at these waves could have attenuated associations with depressive symptoms if respondents moved to neighbourhoods with qualitatively different social and economic conditions. Although residential census tracts are standard units of analyses in studies of neighbourhood exposures, individuals' daily lives may span multiple census tracts beyond their residences. There may also be substantial sociodemographic segregation within tracts that our measures did not capture.

Given the design strengths of our study-nationally representative and diverse sample, repeated assessments of both neighbourhood characteristics and depressive symptoms, and reliable measures of depression-it is tempting to interpret our results as suggesting that, at least on a national level and among adolescents and emerging adults, neighbourhood social and economic characteristics are not associated with mental health. Nevertheless, the potential weaknesses noted above and generally discounted in the aggregate may have obscured real but small effects. The public health implications of putative neighbourhood effects are important because they can have diffuse impacts over large numbers of individuals and because of the substantial burden attributable to depression. ${ }^{54}$ Therefore, we offer the following suggestions for strengthening future studies.

Future attempts to resolve inconsistent findings concerning the role of neighbourhood disadvantage in the risk of depression across the life course will benefit from incorporating prospective designs spanning multiple developmental phases, particularly the highest-risk periods of adolescence and early adulthood. ${ }^{21} 22$ Future studies might also consider both objective and subjective neighbourhood measures and accessibility of services and amenities such as green space that might mitigate deleterious effects of neighbourhood disadvantage. ${ }^{55}$ Multiple statistical approaches could be utilised, including spatial analyses that take conditions of nearby neighbourhoods into account and provide finer-grained characterisation of respondents' neighbourhoods. Clarifying the potential mental health risks associated with neighbourhood disadvantage, including their developmental phase specificity, and identifying neighbourhood-level targets for intervention will ultimately benefit efforts toward optimising the mental health of adolescents and emerging adults.

What is already known on this subject

- Neighbourhood social and economic disadvantage, social fragmentation and income inequality have been associated with depression in some but not all studies.

- Few prospective follow-up studies of neighbourhood effects on depression have been conducted during adolescence when depression tends to emerge, few have utilised nationally representative samples and few have obtained repeated measures of neighbourhood characteristics.

\section{What this study adds}

- We detected no associations of neighbourhood disadvantage with depressive symptoms from mid-adolescence into emerging adulthood despite a prospective design, nationally representative US sample and repeated measures of both neighbourhood disadvantage and depressive symptoms.

- Neighbourhood effects on depression may be too small to detect in geographically dispersed samples of adolescents and young adults.

- Future research should consider the developmental timing of neighbourhood effects, assess both objective and subjective neighbourhood measures, and utilise multiple analytic approaches, including spatial techniques that account for conditions of nearby neighbourhoods and provide a more refined characterisation of individual respondents' neighbourhood exposures. 


\section{Author affiliations}

'Social and Behavioral Sciences Branch, Division of Intramural Population Health Research, Eunice Kennedy Shriver National Institute of Child Health and Human Development, Bethesda, Maryland, USA

${ }^{2}$ Department of Tropical Medicine, Medical Microbiology, and Pharmacology, John A Burns School of Medicine, University of Hawaii at Manoa, Honolulu, Hawaii, USA ${ }^{3}$ Department of Medical and Clinical Psychology, Suicide Care, Prevention and Research Initiative, Uniformed Services University of the Health Sciences, Bethesda, Maryland, USA

${ }^{4}$ Biostatistics Branch, Epidemiology and Biostatistics Program, Division of Cancer Epidemiology and Genetics, National Cancer Institute, Bethesda, Maryland, USA ${ }^{5}$ Department of Mental Health, Johns Hopkins Bloomberg School of Public Health, Baltimore, MD, USA

Acknowledgements The authors are grateful for the expert data management and statistical programming support of Ms. Gina Ma.

Contributors Study concept and design: RBG, AKL and SEG. Drafting of the manuscript: RBG. Acquisition, analysis or interpretation of data; critical revision of the manuscript for important intellectual content; approval of submission and accountability for all aspects of the work in ensuring that any questions related to the accuracy or integrity of any part of the work are appropriately investigated and resolved: All authors.

Funding This research (contract number HHSN275201200001I) was supported in part by the Intramural Research Program of the Eunice Kennedy Shriver National Institute of Child Health and Human Development, and the National Heart, Lung and Blood Institute, the National Institute on Alcohol Abuse and Alcoholism, and Maternal and Child Health Bureau of the Health Resources and Services Administration, with supplemental support from the National Institute on Drug Abuse.

Disclaimer The views, opinions and assertions expressed in this report are those of the authors and should not be construed to represent the views or the official policy or position of any of the sponsoring organisations or agencies or the US government, including the Uniformed Services University or the Department of Defense.

\section{Competing interests None declared.}

Patient consent for publication Not required.

Provenance and peer review Not commissioned; externally peer reviewed.

Data sharing statement Data from the Next Generation Health Study are available through the Data and Specimen Sharing Hub (DASH), a centralized data resource for researchers to access data from research studies funded by the Eunice Kennedy Shriver National Institute of Child Health and Human Development for use in secondary research. For further information, please visit: https://dash.nichd.nih. gov/

\section{REFERENCES}

1 Diez Roux AV, Mair C. Neighborhoods and health. Ann N Y Acad Sci 2010;1186:125-45.

2 Miles R, Coutts C, Mohamadi A. Neighborhood urban form, social environment, and depression. J Urban Health 2012;89:1-18.

3 Richardson R, Westley T, Gariépy G, et al. Neighborhood socioeconomic conditions and depression: a systematic review and meta-analysis. Soc Psychiatry Psychiatr Epidemiol 2015;50:1641-56.

4 Mair C, Diez Roux AV, Galea S. Are neighbourhood characteristics associated with depressive symptoms? A review of evidence. J Epidemiol Community Health 2008;62:940-6.

5 Paczkowski MM, Galea S. Sociodemographic characteristics of the neighborhood and depressive symptoms. Curr Opin Psychiatry 2010;23:337-41.

6 Fagg J, Curtis S, Stansfeld SA, et al. Area social fragmentation, social support for individuals and psychosocial health in young adults: evidence from a national survey in England. Soc Sci Med 2008;66:242-54.

7 Flouri E, Midouhas E, Joshi $H$, et al. Neighbourhood social fragmentation and the mental health of children in poverty. Health Place 2015;31:138-45.

8 Ivory VC, Collings SC, Blakely T, et al. When does neighbourhood matter? multilevel relationships between neighbourhood social fragmentation and mental health. Soc Sci Med 2011;72:1993-2002.

9 Li LW, Xu H, Zhang Z, et al. An ecological study of social fragmentation, socioeconomic deprivation, and suicide in rural China: 2008-2010. SSM Popul Health 2016;2:365-72

10 Pearson AL, Ivory V, Breetzke G, et al. Are feelings of Peace or depression the drivers of the relationship between neighbourhood social fragmentation and mental health in Aotearoa/New Zealand? Health Place 2014;26:1-6.

11 Galea S, Ahern J, Nandi A, et al. Urban neighborhood poverty and the incidence of depression in a population-based cohort study. Ann Epidemiol 2007;17:171-9.
12 Pabayo R, Dunn EC, Gilman SE, et al. Income inequality within urban settings and depressive symptoms among adolescents. J Epidemiol Community Health 2016;70:997-1003.

13 Blakely TA, Kennedy BP, Glass R, et al. What is the lag time between income inequality and health status? J Epidemiol Community Health 2000;54:318-9.

14 Kawachi I, Kennedy BP. Income inequality and health: pathways and mechanisms. Health Serv Res 1999;34:215-27.

15 Kahn RS, Wise PH, Kennedy BP, et al. State income inequality, household income, and maternal mental and physical health: cross sectional national survey. BMJ 2000;321:1311-5.

16 Pabayo R, Kawachi I, Gilman SE. Income inequality among American states and the incidence of major depression. J Epidemiol Community Health 2014;68:110-5.

17 Blair A, Ross NA, Gariepy G, et al. How do neighborhoods affect depression outcomes? A realist review and a call for the examination of causal pathways. Soc Psychiatry Psychiatr Epidemiol 2014;49:873-87.

18 Airaksinen J, Hakulinen C, Elovainio M, et al. Neighborhood effects in depressive symptoms, social support, and mistrust: longitudinal analysis with repeated measurements. Soc Sci Med 2015;136-137:10-16.

19 Barr PB. Early neighborhood conditions and trajectories of depressive symptoms across adolescence and into adulthood. Adv Life Course Res 2018:35:57-68.

20 Dupéré V, Leventhal T, Vitaro F. Neighborhood processes, self-efficacy, and adolescent mental health. J Health Soc Behav 2012;53:183-98.

21 Colman I, Ataullahjan A. Life course perspectives on the epidemiology of depression. Can J Psychiatry 2010;55:622-32.

22 Moffitt TE, Caspi A, Taylor A, et al. How common are common mental disorders? Evidence that lifetime prevalence rates are doubled by prospective versus retrospective ascertainment. Psychol Med 2010;40:899-909.

23 Buu A, Wang W, Wang J, et al. Changes in women's alcoholic, antisocial, and depressive symptomatology over 12 years: a multilevel network of individual, familial, and neighborhood influences. Dev Psychopathol 2011;23:325-37.

24 Clark C, Ryan L, Kawachi I, et al. Witnessing community violence in residential neighborhoods: a mental health hazard for urban women. J Urban Health 2008;85:22-38.

25 Conway KP, Vullo GC, Nichter B, et al. Prevalence and patterns of polysubstance use in a nationally representative sample of 10 th graders in the United States. J Adolesc Health 2013;52:716-23.

26 Dunn EC, Johnson RM, Green JG. The modified depression scale (MDS): a brief, nocost assessment tool to estimate the level of depressive symptoms in students and schools. School Ment Health 2012;4:34-45.

27 Goldstein AL, Walton MA, Cunningham RM, et al. Violence and substance use as risk factors for depressive symptoms among adolescents in an urban emergency department. J Adolesc Health 2007;40:276-9.

28 Wang J, lannotti RJ, Luk JW, et al. Co-occurrence of victimization from five subtypes of bullying: physical, verbal, social exclusion, spreading rumors, and cyber. J Pediatr Psychol 2010;35:1103-12.

29 Pilkonis PA, Choi SW, Reise SP, et al. Item banks for measuring emotional distress from the patient-reported outcomes measurement information system (PROMIS $R$ ): depression, anxiety, and anger. Assessment 2011;18:263-83.

30 Irwin DE, Stucky B, Langer MM, et al. An item response analysis of the pediatric PROMIS anxiety and depressive symptoms scales. Qual Life Res 2010;19:595-607.

31 Varni JW, Magnus B, Stucky BD, et al. Psychometric properties of the PROMIS ¿ pediatric scales: precision, stability, and comparison of different scoring and administration options. Qual Life Res 2014;23:1233-43.

32 Applied Geographic Solutions. CrimeRisk, 2018. Available: AGS-CrimeRiskMethodology-2018.pdf

33 Choi SW, Schalet B, Cook KF, et al. Establishing a common metric for depressive symptoms: linking the BDI-II, CES-D, and PHQ-9 to PROMIS depression. Psychol Assess 2014;26:513-27.

34 Olino TM, Yu L, McMakin DL, et al. Comparisons across depression assessment instruments in adolescence and young adulthood: an item response theory study using two linking methods. J Abnorm Child Psychol 2013;41:1267-77.

35 Blebu BE, Ro A, Kane JB, et al. An examination of preterm birth and residential social context among black immigrant women in California, 2007-2010. Journal of Community Health 2018;86.

36 Cohen-Cline H, Beresford SAA, Barrington WE, et al. Associations between neighbourhood characteristics and depression: a twin study. J Epidemiol Community Health 2018;72:202-7.

37 Cole H, Duncan DT, Ogedegbe G, et al. Neighborhood socioeconomic disadvantage; neighborhood racial composition; and hypertension stage, awareness, and treatment among hypertensive black men in New York City: does nativity matter? J Racial Ethn Health Disparities 2016. doi:10.1007/s40615-016-0289-x

38 DeCuir J, Lovasi GS, El-Sayed A, et al. The association between neighborhood socioeconomic disadvantage and high-risk injection behavior among people who inject drugs. Drug Alcohol Depend 2018;183:184-91.

39 Tsui J, Gee GC, Rodriguez HP, et al. Exploring the role of neighborhood sociodemographic factors on HPV vaccine initiation among low-income, ethnic minority girls. J Immigr Minor Health 2013;15:732-40. 
40 United States Bureau of the Census. American community survey (ACS): design and methodology report. Washington DC: United States Department of Commerce, 2018.

41 Pabayo R, Belsky J, Gauvin L, et al. Do area characteristics predict change in moderate-to-vigorous physical activity from ages 11 to 15 years? Soc Sci Med 2011;72:430-8.

42 Fontenot KS, Semega J, Kollar M J, et al. Income and poverty in the United States: 2017. Washington DC: U S Government Printing Office, 2018.

43 Ceballo R, McLoyd VC. Social support and parenting in poor, dangerous neighborhoods. Child Dev 2002;73:1310-21.

44 Crosier T, Butterworth P, Rodgers B. Mental health problems among single and partnered mothers. The role of financial hardship and social support. Soc Psychiatry Psychiatr Epidemiol 2007;42:6-13.

45 Reboussin BA, lalongo NS, Green KM, et al. The impact of the urban neighborhood environment on marijuana trajectories during emerging adulthood. Prevention Science 2018.

46 Glynn K, Maclean H, Forte T, et al. The association between role overload and women's mental health. J Womens Health 2009;18:217-23.

47 Sampson RJ, Raudenbush SW, Earls F. Neighborhoods and violent crime: a multilevel study of collective efficacy. Science 1997;277:918-24.

48 Sampson RJ. Collective regulation of adolescent misbehavior: validation results from eighty Chicago neighborhoods. Journal of Adolescent Research 1997; 12:227-44.
49 Organisation for Economic Co-Operation and Development. Glossary of statistical terms. Geneva Switzerland: Organisation for Economic Co-operation and Development, 2006

50 United States Bureau of the Census. Comparing 2006-2010 ACS 5-year and 20112015 ACS 5-year estimates. Washington, DC: United States Bureau of the Census, 2015.

51 Currie C, Molcho M, Boyce W, et al. Researching health inequalities in adolescents: the development of the health behaviour in school-aged children (HBSC) family affluence scale. Soc Sci Med 2008;66:1429-36.

52 Stirling K, Toumbourou JW, Rowland B. Community factors influencing child and adolescent depression: a systematic review and meta-analysis. Aust N Z J Psychiatry 2015;49:869-86.

53 Telzer EH, van Hoorn J, Rogers CR, et al. Social influence on positive youth development: a developmental neuroscience perspective. Adv Child Dev Behav 2018;54:215-58.

54 GBD 2015 DALYs and HALE Collaborators. Global, regional, and national disabilityadjusted life-years (DALYs) for 315 diseases and injuries and healthy life expectancy (HALE), 1990-2015: a systematic analysis for the global burden of Disease Study 2015. Lancet 2016:388:1603-58.

55 Gariepy G, Blair A, Kestens Y, et al. Neighbourhood characteristics and 10-year risk of depression in Canadian adults with and without a chronic illness. Health Place 2014;30:279-86. 